\title{
Towards Accurate State of Charge Estimation for Lithium-ion Batteries using Self-supervised Transformer Model: A Deep Learning Approach
}

M Hannan ( $\nabla$ hannan@uniten.edu.my )

Universiti Tenaga Nasional

Dickson How

Universiti Tenaga Nasional

M. S. Hossain Lipu

Universiti Kebangsaan Malaysia

M Mansor

Universiti Tenaga Nasional

Pin Ker

Universiti Tenaga Nasional

Z Dong

UNSW Sydney

K Sahari

Universiti Tenaga Nasional

$S$ Tiong

Universiti Tenaga Nasional

Kashem Muttaqi

University of Wollongong

T M I Mahlia

University of Technology Sydney

Fred Blaabjerg

Aalborg University

\section{Research Article}

Keywords: state of charge, transformer model, deep learning, transfer learning, self-supervised learning, Li-ion,data-driven

Posted Date: July 8th, 2021

DOI: https://doi.org/10.21203/rs.3.rs-687515/v1 
License: (c) (i) This work is licensed under a Creative Commons Attribution 4.0 International License. Read Full License 


\title{
Towards accurate state of charge estimation for lithium-ion batteries using self-supervised transformer model: A deep learning approach
}

\author{
M A Hannan ${ }^{1}$, Dickson N T How ${ }^{1}$, M S Hossain Lipu $^{2}$, M. Mansor ${ }^{1}$, Pin Jern Ker ${ }^{3}$, Z Y Dong ${ }^{4}$, K S M Sahari ${ }^{5}$, S K \\ Tiong $^{3}$, K M Muttaqi ${ }^{6}, \mathrm{~T}^{\mathrm{M}}$ Indra Mahlia ${ }^{7}, \mathrm{~F}_{\text {Blaabjerg }}^{8}$ \\ ${ }^{1}$ Department of Electrical Power Engineering, COE, Universiti Tenaga Nasional, Kajang 43000, Malaysia \\ ${ }^{2}$ Dept of Electrical, Electronic and Systems Engineering, Universiti Kebangsaan Malaysia, Bangi 43600, Malaysia \\ ${ }^{3}$ Institute of Sustainable Energy, Universiti Tenaga Nasional, Kajang 43000, Malaysia \\ ${ }^{4}$ School of Electrical Engineering and Telecommunications, UNSW, Kensington NSW 2033, Australia \\ ${ }^{5}$ Department of Mechanical Engineering, COE, Universiti Tenaga Nasional, Kajang 43000, Malaysia \\ ${ }^{6}$ School of Electrical, Computer and Telecommunications Engineering, University of Wollongong, NSW 2522, \\ Australia \\ ${ }^{7}$ School of Information, Systems and Modelling, University of Technology Sydney, Ultimo NSW 2007, Australia \\ ${ }^{8}$ Department of Energy Technology, Aalborg University, 9220 Aalborg, Denmark
}

*Corresponding author's email: hannan@uniten.edu.my; dickson@uniten.edu.my

\begin{abstract}
Accurate state of charge (SOC) estimation of lithium-ion (Li-ion) batteries is crucial in prolonging cell lifespan and ensuring its safe operation for electric vehicle applications. In this article, we propose the deep learning-based transformer model trained with self-supervised learning (SSL) for end-to-end SOC estimation without the requirements of feature engineering or adaptive filtering. We demonstrate that with the SSL framework, the proposed deep learning-enabled transformer model achieves the lowest root-mean-square-error (RMSE) of $1.2 \%$ and a mean-absolute-error (MAE) of $0.7 \%$ on the test dataset at various ambient temperatures. With SSL, the proposed model can be trained with as few as 5 epochs using only $20 \%$ of the total training data and still achieves less than $1.9 \%$ RMSE on the test data. Finally, we also demonstrate that the learning weights during the SSL training can be transferred to a new Li-ion cell with different chemistry and still achieve on-par performance compared to the models trained from scratch on the new cell.
\end{abstract}

Keywords: state of charge, transformer model, deep learning, transfer learning, self-supervised learning, Li-ion, data-driven. 


\section{Introduction}

The transportation and electricity production sectors account for more than $50 \%$ of total green-house gas emissions ${ }^{1}$ as both rely on fossil fuels as the energy source. Promising solutions are the electrification of the transportation industry and the decarbonization of electrical grids ${ }^{2,3}$. However, the mass adoption of electric vehicles and renewable energy remains low due to the high adoption cost which can be attributed to the Li-ion batteries $^{4}$. A major challenge in Li-ion batteries research is the state of charge (SOC) estimation which signifies the amount of charge left in a Li-ion battery cell ${ }^{5}$. Accurate SOC estimation allows the Li-ion battery cells to be used to its maximum potential before disposal, resulting in tremendous cost savings in the manufacturing and adoption costs ${ }^{6}$. Nevertheless, it is a notoriously hard to quantify SOC as it cannot be practically measured by sensors outside laboratory environment with existing sensor technologies ${ }^{7}$.

Two most common approaches used in SOC estimation are the model-based and data-driven approaches ${ }^{8}$. Model-based approach leverages on an in-depth understanding of domain knowledgesuch as the internal chemical reaction in the cell, electrical properties of the components used to model them and complex mathematical equations to model the $\mathrm{SOC}^{9}$. Prominent model-based techniques include the Sliding Mode Observer ${ }^{10}$, Luenberger Observer $^{11}$ and Kalman filters ${ }^{12}$. While model-based approach can result in reliable and accurate models, it requires an extensive domain knowledge, rigorous feature engineering, and requires relatively long development time ${ }^{13}$. Apart from that, model-based approach also does not scale well across battery cells different chemistry. As a result, alterations in cell chemistry requires a re-development of the model ${ }^{14}$. Additionally, model-based approach also does not account for anomalies in cells such as manufacturing inconsistencies, unpredictable operating conditions, cell degradation, and so forth ${ }^{15}$. Due to these shortcomings, more researchers are shifting their attention to using the data-driven approach for SOC estimation. In this approach, the SOC is directly modeled from observable signals such as voltage, current and temperature of the Li-ion battery cell sampled over diverse operating conditions across different cell chemistry and manufacturers ${ }^{16}$. One method that has been gaining traction lately is the use of a data-driven technique known as deeplearning (DL) ${ }^{17}$. DL has great potential for SOC estimation due to its powerful capability to learn any function given the right data according to the universal approximation theorem $^{18}$. In essence, DL can be used to directly approximate the relationship between the measurable cell signals (voltage, current, temperature) and the SOC with no additional processing such as using adaptive filters ${ }^{19}$. This eliminates the needs of manual feature engineering which can take a considerable amount of time and expert domain knowledge and still produce accurate SOC estimation results. Pioneering works by authors in ${ }^{20}$ and Chemali et al. ${ }^{21}$ introduce long short-term memory (LSTM) and deep neural network DNN to directly estimate SOC from cell voltage, current and temperature with no additional filters. The proposed model achieved lowest MAE of $2.17 \%$ over a varying ambient temperature dataset. Authors in ${ }^{22,23}$ proposed the utilization of gated recurrent unit (GRU) model to directly estimate SOC over a wide ambient temperature range with a RMSE of $3.5 \%$ under untrained temperature. There are also authors who proposed deep convolutional models such as in ${ }^{24-26}$ with approximately less than 3\% MAE on untrained data. Nonetheless, there are works on hybridizing convolutional and recurrent models such as ${ }^{27,28}$ with under $2 \%$ RMSE on varying ambient temperature.

However, there exist several research gaps with the existing DL methods for SOC estimation. Firstly, all the cited works uses the supervised learning (SL) scheme to train the models which is known to require massive amount of data to accomplish ${ }^{29}$. Even in the scenarios where adequate data is available the training time for DL models typically takes many hours or days to complete ${ }^{20}$. Secondly, the models that are trained on one cell chemistry do not apply to other cell chemistry. Even though preliminary work indicates that the transfer learning is possible ${ }^{24}$, further tests are still required to verify its accuracy if it applies to more cell with differing chemistry. In most cases a model that is trained on one Li-ion battery cell data does not generalize well across anothercell and may require re-training of the model from scratch. Thirdly, most DL models use the recurrent DL architecture which may prove to work well with sequence data such as the SOC but are hard and slow to train ${ }^{30}$. Recurrent models also do not leverage on the parallel GPU computation that could significantly improve training time ${ }^{31}$. Lastly, even though recurrent architectures such as the LSTM or GRU can handle long sequences fairly well, they are still susceptible to vanishing gradient for longer sequences ${ }^{32}$. Due to these limitations, recurrent models are generally superseded by another architecture known as the Transformer in many domains such as computer vision ${ }^{33,34}$ and natural language processing ${ }^{35,36}$.

In this article, we introduce a new DL architecture for SOC estimation known as the Transformer. Apart from that, we propose a training framework that leverages on self-supervised learning (SSL) ${ }^{29,37}$ and make it possible to train the Transformer on scarce amounts of Li-ion data ina short time and achieve higher estimation accuracy compared to models trained with conventional fully-supervised method. With the proposed framework, we demonstrate that the learned parameters from one cell can be transferred to another by fine-tuning the model on little data with very short training time (approximately 30 minutes on a GPU). This proposed framework also incorporates various recent DL techniques such as using Ranger optimizer with learning rate finder, time series data augmentation, and Log-Cosh loss function to boost the accuracy of the Transformer. Finally, we conclude 
the study by comparing and validating the performance of our proposed model to other recent DL architectures for SOC estimation. The key contributions of this study are as follows:

- We introduce the transformer DL architecture for end-to-end SOC estimation with no feature engineering or adaptive filtering.

- We propose the SSL training framework to train the proposed architecture in a very short amount of time and achieves improved estimation accuracy compared to conventional training framework.

- The proposed model's parameters are transferable to a different cell type and requires only five training epochs to achieve RMSE $\leq 2.5 \%$.

- The SSL training framework enables the proposed model to be re-trained with as few as $20 \%$ of the total training data and still achieve lower error compared to the models that do not use the SSL framework.

- We evaluate and validate the performance of the proposed model to other state-of-the-art DL architectures for SOC estimation.

\section{Results}

In the first subsection, we highlight the estimation accuracy of the proposed model trained at room temperature and varying ambient temperatures and compare the estimation robustness against other DL models. In the subsequent section, we study the influence of pre-training on the model and show that the pre-trained model outperforms the non-pre-trained models in estimation accuracy and convergence time. Additionally, we highlight that the learned weights of the pre-trained model can be transferred to perform estimation on a different cell with different chemistry with short re-training or fine-tuning.

Estimation accuracy under constant ambient temperature. In this section, we demonstrate the estimation accuracy and efficacy of the proposed model based on data sampled at room temperature. The error metrics of all models are tabulated in Table 1, sorted in ascending order of the test error. We found that the proposed model performance on SOC estimation accuracy of the RMSE in terms of training, validation and testing are $1.1087 \%, 0.8661 \%, 0.9056 \%$ and the MAE are $0.3289 \%, 0.4059 \%$, and $0.4459 \%$, respectively. It is observed that the proposed model outperforms all other models on the test dataset of RMSE and MAE tabulated in Table 1. For comparison, a baseline Transformer model that was trained using conventional training framework is included. Results indicate that the baseline model only scores abysmally compared to other models except the DNN, suggestingthat the training framework plays a pivotal role in the robust performance of the proposed model. We observe that the recurrent models (GRU and LSTM) outperformed the ir convolutional and hybrid counterpart, which is not surprising as the recurrent models are specifically designed to handle sequence data well. Both the GRU and LSTM were configured to use single hidden layer with 100 neurons. Despite the compromise in estimation accuracy, convolutional models may be advantageous in training complexity compared to the recurrent models, as they are relatively easier to train and could better utilize GPU parallelization. The Resnet model is based on the Residual Network architecture ${ }^{38}$ adapted to work sequential data ${ }^{39}$. Inception Time is based on the implementation in ${ }^{40}$ which has shown superior benchmark performance in sequential data. ResCNN consist of convolutional neural network with Residual blocks as inputs. FCN consist of only convolution operation with no pooling operations has shown promising performance in ${ }^{41}$. GRU-FCN and LSTM-FCN are the hybrid models combining recurrent and convolutional models to obtain the advantages of both. The estimation plot on the test dataset consisting of US06, LS92 and UDDS drive cycle is shown in Fig. 1.

Table 1. SOC estimation accuracy comparison on various DL models on the train, validation, and test dataset.

\begin{tabular}{|c|c|c|c|c|c|c|c|}
\hline \multirow[t]{2}{*}{ Name } & \multirow[t]{2}{*}{ Arch. Type } & \multicolumn{2}{|c|}{ Train Error $(\%)$} & \multicolumn{2}{|c|}{ Valid. Error (\%) } & \multicolumn{2}{|c|}{ Test Error (\%) } \\
\hline & & RMSE & MAE & RMSE & MAE & RMSE & MAE \\
\hline Proposed & Transformer & 1.1087 & 0.3289 & 0.8661 & 0.4059 & 0.9056 & 0.4459 \\
\hline GRU & Recurrent & 1.5028 & 0.4461 & 0.9852 & 0.3624 & 1.0686 & 0.4877 \\
\hline LSTM & Recurrent & 1.6951 & 0.5404 & 1.0699 & 0.4370 & 1.1381 & 0.5341 \\
\hline Resnet & Convolutional & 1.5523 & 0.5647 & 1.1395 & 0.5924 & 1.3349 & 0.7859 \\
\hline ResCNN & Convolutional & 1.6121 & 0.6134 & 1.1879 & 0.6548 & 1.3454 & 0.8031 \\
\hline InceptionTime & Convolutional & 1.8177 & 0.7538 & 1.3079 & 0.7259 & 1.4404 & 0.8202 \\
\hline GRU-FCN & Hybrid & 1.7138 & 0.5978 & 1.3405 & 0.6939 & 1.4477 & 0.8228 \\
\hline $\mathrm{FCN}$ & Convolutional & 1.8635 & 0.7685 & 1.2912 & 0.6732 & 1.5555 & 0.9642 \\
\hline LSTM-FCN & Hybrid & 1.7619 & 0.7611 & 1.4123 & 0.8218 & 1.7771 & 1.1954 \\
\hline Baseline & Transformer & 3.8265 & 2.2195 & 2.4050 & 1.2735 & 3.6116 & 2.7453 \\
\hline DNN & Feedforward & 11.0576 & 8.6442 & 10.4213 & 8.5476 & 10.1347 & 8.2181 \\
\hline
\end{tabular}




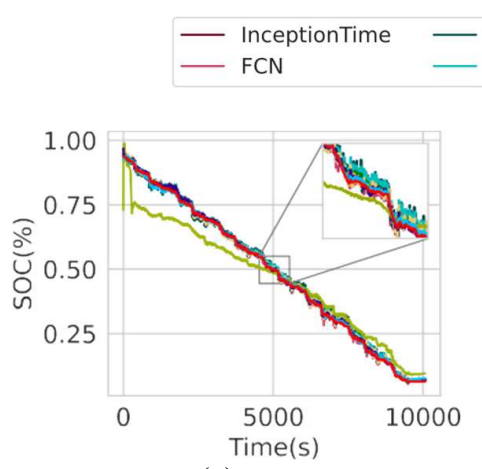

(a)

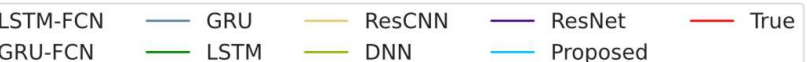

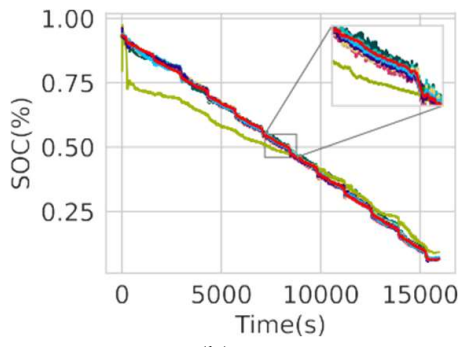

(b)

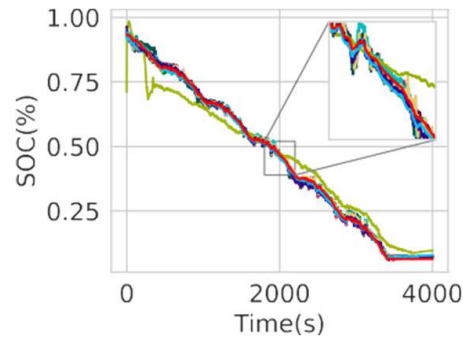

(c)

Fig. 1 SOC estimation at room temperature. (a) LA92 drive cycle at $25^{\circ} \mathrm{C}$. (b) UDDS drive cycle at $25^{\circ} \mathrm{C}$. (c) US06 drive cycle at $25^{\circ} \mathrm{C}$.

Estimation accuracy under varying ambient temperatures. In this subsection, we compare the estimation accuracy of the proposed model to other widely used DL models of various architectures. Among all DL architectures compared in the study, the proposed transformer model achieved the lowest RMSE of $1.1075 \%$, $1.3139 \%$ and $1.1914 \%$ and MAE of $0.4441 \%, 0.5680 \%$ and $0.6502 \%$ on the test drive cycles outperforming even the recurrent models which has been widely used for SOC estimation as shown in Table 2. We also note that the convolutional models such as the Resnet ${ }^{41}$ and the Inception Time ${ }^{40}$ also outperformed the conventional GRU ${ }^{42}$ and $\mathrm{LSTM}^{43}$ model. The baseline Transformer model that is not trained with the proposed training framework scores poorly along with the feedforward DNN. Fig. 2 and Fig. 3 illustrate the SOC estimation plots of the proposed model across all test drive cycles at above and below zero ${ }^{\circ} \mathrm{C}$ ambient temperature, respectively. Traditionally, SOC estimation under low ambient temperature settings are extremely challenging due to the difference in the dynamics of chemical reactions in the cell ${ }^{44}$. However, observation in the estimated SOC by the proposed Transformer model shows promising results indicating its robustness in estimating SOC at extreme cold temperatures up to $-20^{\circ} \mathrm{C}$.

Table 2: Cross-comparison of the SOC estimation accuracy on the train, validation, and test dataset at varying ambient temperatures.

\begin{tabular}{|c|c|c|c|c|c|c|c|}
\hline \multirow[t]{2}{*}{ Name } & \multirow[t]{2}{*}{ Arch. Type } & \multicolumn{2}{|c|}{ Train Error (\%) } & \multicolumn{2}{|c|}{ Valid. Error (\%) } & \multicolumn{2}{|c|}{ Test Error (\%) } \\
\hline & & RMSE & MAE & RMSE & MAE & RMSE & MAE \\
\hline Proposed & Transformer & 1.1075 & 0.4441 & 1.3139 & 0.5680 & 1.1914 & 0.6502 \\
\hline Resnet & Convolutional & 1.2510 & 0.5077 & 1.5736 & 0.8058 & 1.3636 & 0.7771 \\
\hline InceptionTime & Convolutional & 1.2112 & 0.4967 & 1.2573 & 0.5985 & 1.3792 & 0.8152 \\
\hline GRU & Recurrent & 1.4183 & 0.3505 & 1.5989 & 0.4901 & 1.3856 & 0.5847 \\
\hline LSTM & Recurrent & 1.5549 & 0.5681 & 1.5121 & 0.5961 & 1.4498 & 0.7300 \\
\hline ResCNN & Convolutional & 1.5803 & 0.7712 & 1.8668 & 0.9860 & 1.5860 & 0.9048 \\
\hline GRU-FCN & Hybrid & 1.5903 & 0.7695 & 2.0070 & 0.9893 & 1.6215 & 0.9269 \\
\hline $\mathrm{FCN}$ & Convolutional & 1.7358 & 0.8879 & 2.1282 & 1.1033 & 1.7808 & 1.0810 \\
\hline LSTM-FCN & Hybrid & 1.9962 & 1.0561 & 2.2292 & 1.1804 & 1.9248 & 1.1552 \\
\hline Baseline & Transformer & 3.7466 & 2.4466 & 4.4805 & 3.4827 & 3.6958 & 2.8129 \\
\hline DNN & Feedforward & 8.9848 & 6.8349 & 8.1916 & 6.1570 & 7.7789 & 6.1567 \\
\hline
\end{tabular}




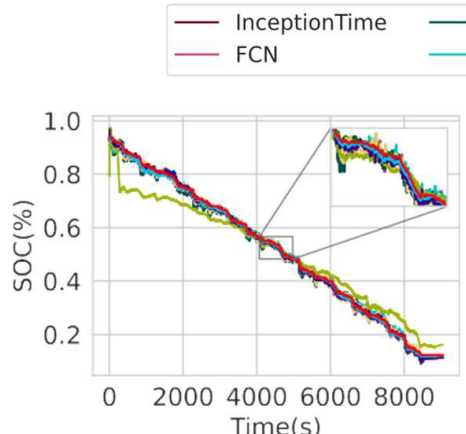

(a)

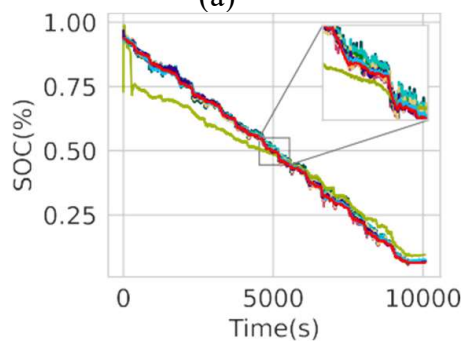

(d)

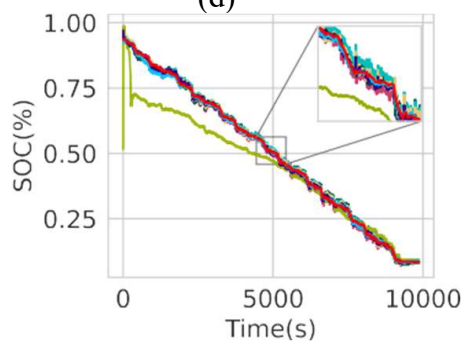

(g)

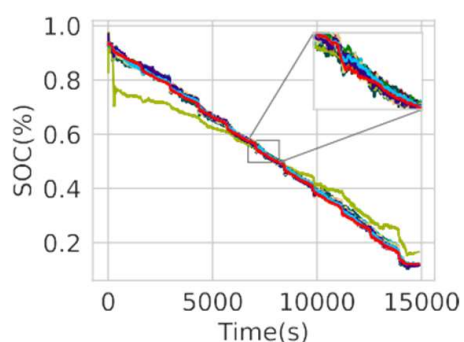

(b)

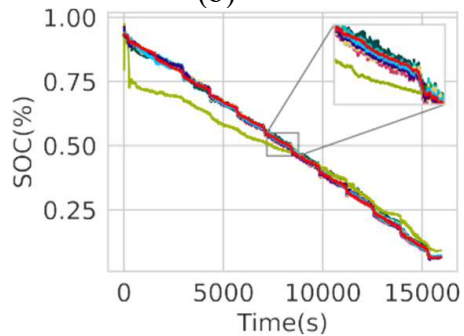

(e)

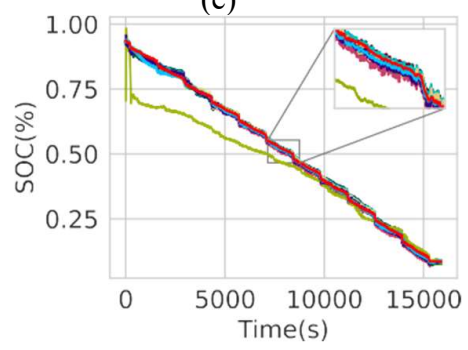

(h)
ResNet
Proposed

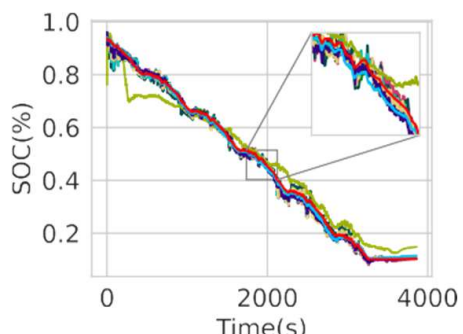

(c)

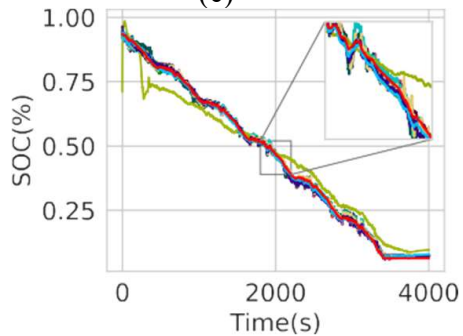

(f)

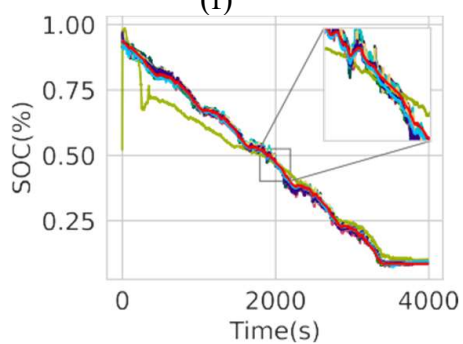

(i)

Fig. 2 SOC estimation at above zero ambient temperatures. (a) LA92 drive cycle at $10^{\circ} \mathrm{C}$. (b) UDDS drive cycle at $10^{\circ} \mathrm{C}$. (c) US06 drive cycle at $10^{\circ} \mathrm{C}$. (d) LA92 drive cycle at $25^{\circ} \mathrm{C}$. (e) UDDS drive cycle at $25^{\circ} \mathrm{C}$. (f) US06 drive cycle at $25^{\circ} \mathrm{C}$. (g) LA92 drive cycle at $40{ }^{\circ} \mathrm{C}$.(h) UDDS drive cycle at $40^{\circ} \mathrm{C}$. (i) US06 drive cycle at $40{ }^{\circ} \mathrm{C}$.

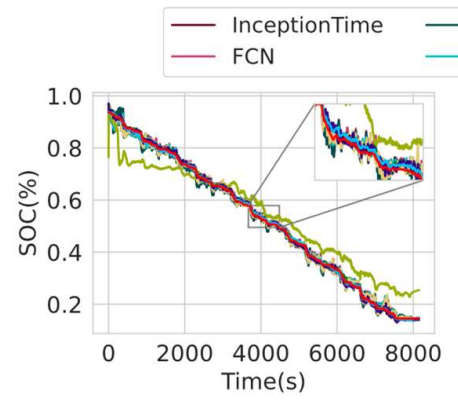

(a)

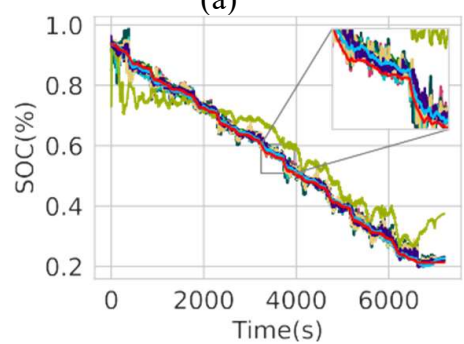

(d)

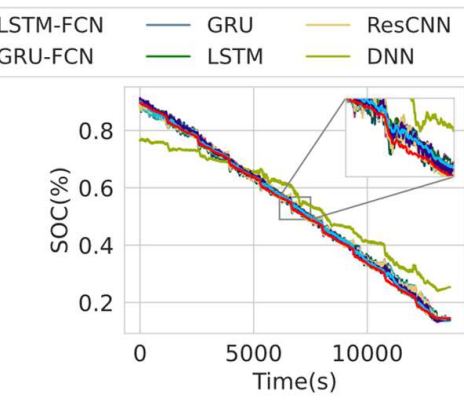

(b)

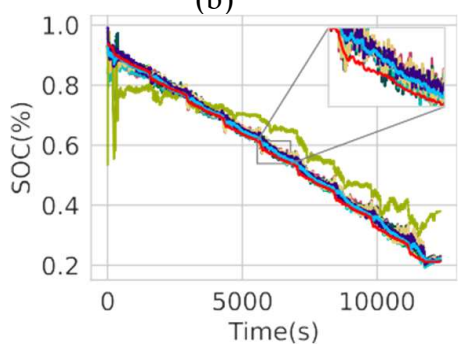

(e)

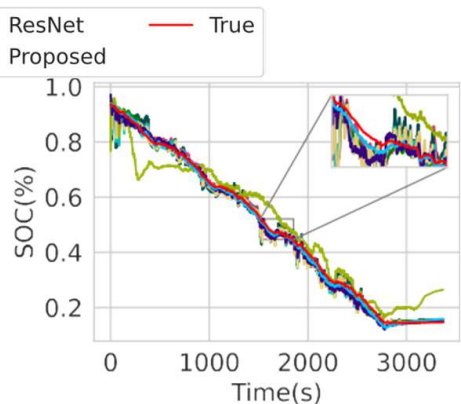

(c)

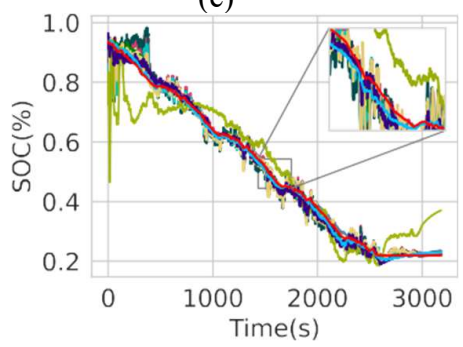

(f) 


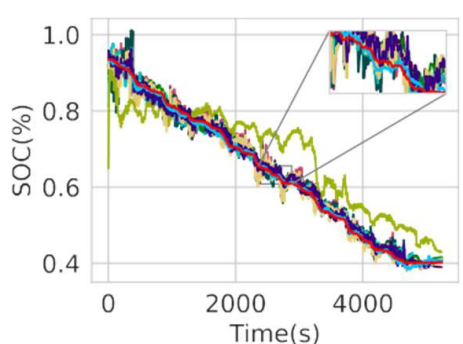

(g)

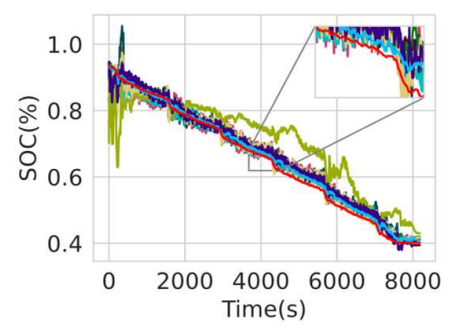

(h)

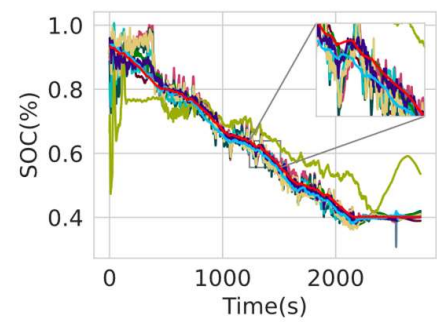

(i)

Fig. 3 SOC estimation at below zero ambient temperatures. (a) LA92 drive cycle at $0 \circ \mathrm{C}$. (b) UDDS drive cycle at $0{ }^{\circ} \mathrm{C}$. (c) US06 drive cycle at $0{ }^{\circ} \mathrm{C}$. (d) LA92 drive cycle at $-10{ }^{\circ} \mathrm{C}$. (e) UDDS drive cycle at $-10^{\circ} \mathrm{C}$. (f) US06 drive cycle at $-10^{\circ} \mathrm{C}$. (g) LA92 drive cycle at $-20^{\circ} \mathrm{C}$.(h) UDDS drive cycle at $-20{ }^{\circ} \mathrm{C}$. (i) US06 drive cycle at $-20 \circ \mathrm{C}$.

\section{Influence of Pre-training}

Size of Training Data. In this section, we investigate the influence of unsupervised pre-training on the amount of required data to train the proposed model with a low error rate. We divided our experiments into three scenarios. In the first scenario, we are pre-trained and re-trained/fine-tuned the model with all (100\%) available training data. The second and third scenarios were with $50 \%$ and $20 \%$ of the training data, respectively. In all scenarios, we noted that the training time and error metrics. All training in this section was only performed for only 5 epochs to illustrate the short amount of training time required to achieve low error rate. There are three modes of training used in this setup namely the pre-training (PT), re-training (RT), fine-tuning (FT), and full training (T). In PT, the model was trained on unlabeled dataset with unsupervised learning. In RT, the mode was trained on a labeled dataset with supervised learning. In FT, the model was frozen except for the last layer and trained with supervised learning. In $\mathrm{T}$, the model was trained from scratch with supervised learning.

Table 3 shows the results obtained. We observe that when we pre-trained and re-trained the model on all available data (row 1), the error metric is lower compared to the model that is not pre-trained (row 3 ) at approximately same duration of training time. We observe that in the event where we pre-trained and fine-tuned the model (row 3), even though the model only updates the weights of the final layer, it still scores a respectable $2.01 \%$ RMSE with a reduction ofabout 10 minutes training time compared to the previous two modes. The effect of pre-training is even more pronounced in the second scenario where we only re-train and fine-tune the modelson $20 \%$ of available data. At approximately the same amount of training time, the pre-trained model (row 4) scores lower on the non-pre-trained model (row 6). In this section we showed that pre-training helps in reducing the test error with approximately same amount of training time especially when data is scarce.

Table 3: The influence of pre-training on the training time, training data amount, and cross-datasetgeneralization.

\begin{tabular}{|c|c|c|c|c|c|c|c|c|}
\hline \multirow[t]{2}{*}{ *Mode } & \multirow{2}{*}{$\begin{array}{l}\text { Train Time } \\
\text { (mins.) }\end{array}$} & \multirow[t]{2}{*}{ Train Data (\%) } & \multicolumn{2}{|c|}{ Train Error (\%) } & \multicolumn{2}{|c|}{ Valid. Error (\%) } & \multicolumn{2}{|c|}{ Test Error (\%) } \\
\hline & & & RMSE & MAE & RMSE & MAE & RMSE & MAE \\
\hline$\overline{\mathrm{PT}+\mathrm{RT}}$ & 33.1 & 100 & 1.4378 & 0.7229 & 1.6207 & 0.8074 & 1.4257 & 0.8384 \\
\hline $\mathrm{PT}+\mathrm{FT}$ & 20.8 & 100 & 2.2281 & 1.4314 & 2.5211 & 1.4666 & 2.1042 & 1.4907 \\
\hline $\mathrm{T}$ & 32.0 & 100 & 1.6981 & 0.9980 & 1.8448 & 1.1185 & 1.6367 & 1.0675 \\
\hline$\overline{\mathrm{PT}+\mathrm{RT}}$ & 15.3 & 50 & 1.4318 & 0.6980 & 1.6749 & 0.9305 & 1.6578 & 1.0609 \\
\hline $\mathrm{PT}+\mathrm{FT}$ & 10.0 & 50 & 2.4540 & 1.6110 & 2.8765 & 1.7509 & 2.3893 & 1.7097 \\
\hline $\mathrm{T}$ & 15.3 & 50 & 2.6117 & 1.7230 & 2.5167 & 1.5975 & 2.3953 & 1.7047 \\
\hline$\overline{\mathrm{PT}+\mathrm{RT}}$ & 6.7 & 20 & 1.3072 & 0.6778 & 2.7138 & 1.3145 & 1.8993 & 1.1944 \\
\hline $\mathrm{PT}+\mathrm{FT}$ & 5.3 & 20 & 2.1391 & 1.4582 & 3.0693 & 1.8374 & 2.7173 & 1.9036 \\
\hline $\mathrm{T}$ & 6.8 & 20 & 2.4656 & 1.5721 & 2.8898 & 1.7170 & 2.5900 & 1.7833 \\
\hline
\end{tabular}

*PT-Pre-training, RT-Re-training, FT-Fine-tuning, T-Training

Transfer Learning. In this section we investigate the role of unsupervised pre-training in helping the model to generalize its estimation capacity across different cell chemistry. We pre-trained the model on the LG 18650 LiNiMnCoO 2 cell and tested the model's estimation capacity on a Panasonic $18650 \mathrm{LiNiCoAlO}_{2}$ cell, similar to the cells used in some Tesla vehicles. Table 4 shows the performance of the proposed model with no changes in the model architecture. 
Table 4 Cross dataset performance.

\begin{tabular}{|c|c|c|c|c|c|c|c|c|c|}
\hline \multirow[t]{2}{*}{ *Mode } & \multirow[t]{2}{*}{ PT Dataset } & \multirow{2}{*}{$\begin{array}{l}\text { RT/FT } \\
\text { Dataset }\end{array}$} & \multirow{2}{*}{$\begin{array}{l}\text { Train Data } \\
(\%)\end{array}$} & \multicolumn{2}{|c|}{ Train Error $(\%)$} & \multicolumn{2}{|c|}{ Valid. Error (\%) } & \multicolumn{2}{|c|}{ Test Error (\%) } \\
\hline & & & & RMSE & MAE & RMSE & MAE & RMSE & MAE \\
\hline$\overline{\mathrm{PT}+\mathrm{RT}}$ & LG & Panasonic & 100 & 1.3128 & 0.7948 & 1.4245 & 0.8266 & 2.3558 & 1.5477 \\
\hline $\mathrm{PT}+\mathrm{FT}$ & LG & Panasonic & 100 & 1.9400 & 1.2514 & 2.4220 & 1.4254 & 3.3119 & 2.3490 \\
\hline $\mathrm{T}$ & - & Panasonic & 100 & 1.2744 & 0.8086 & 1.4597 & 0.9165 & 2.7054 & 2.0383 \\
\hline$\overline{\mathrm{PT}+\mathrm{RT}}$ & LG & Panasonic & 50 & 1.1674 & 0.6602 & 1.5817 & 0.9911 & 3.0465 & 2.1906 \\
\hline $\mathrm{PT}+\mathrm{FT}$ & LG & Panasonic & 50 & 2.0930 & 1.3643 & 2.9021 & 1.7583 & 3.8216 & 2.6578 \\
\hline$\underline{T}$ & - & Panasonic & 50 & 1.4121 & 0.9128 & 1.8295 & 1.1583 & 3.1793 & 2.3900 \\
\hline$\overline{\mathrm{PT}+\mathrm{RT}}$ & LG & Panasonic & 20 & 1.2377 & 0.7689 & 2.6921 & 2.0752 & 3.7735 & 2.8997 \\
\hline $\mathrm{PT}+\mathrm{FT}$ & LG & Panasonic & 20 & 1.9597 & 1.2957 & 3.4970 & 2.4140 & 3.8079 & 2.6289 \\
\hline $\mathrm{T}$ & - & Panasonic & 20 & 1.5928 & 1.0598 & 3.1262 & 2.0098 & 4.3440 & 2.6932 \\
\hline$\overline{\mathrm{PT}+\mathrm{RT}}$ & LG & LG & 100 & 9.4881 & 8.6845 & 9.7792 & 8.9473 & 11.3084 & 10.4053 \\
\hline$\underline{\mathrm{PT}+\mathrm{RT}}$ & Panasonic & Panasonic & 100 & 1.0752 & 0.5757 & 1.3006 & 0.7778 & 2.3462 & 1.6941 \\
\hline
\end{tabular}

*PT-Pre-training, RT-Re-training, FT-Fine-tuning, T-Training

We divided our test into three testing scenarios. In the first scenario, we pre-trained the model on the LG cell and tested the model on Panasonic cell with $100 \%$ the amount of training data available as shown in the first three rows. In the second scenario we performed the same routine as the first but this time with a reduced amount of training data. Only $20 \%$ of the total data was used in this test group. In the third scenario we pre-trained and retrained the model on the same cell type with all available data. Unsurprisingly, the best performing mode is when the model pre-trained and re-trained on the Panasonic cell and the worst performing model is when the model was pre-trained and re-trained on the LG cell. However, when the model was pre-trained on the LG cell and retrained on the Panasonic cell, the test error rate is almost on par with the best performing mode. This suggests that the pre-training helps in downstream re-training despite the difference in cell type. In the scenario when the model was trained on less data ( $20 \%$ of the training data), we observe that without pre-training (row 6$)$ the model yields high test errors. In rows 4 and 5, we observe that the error rate is reduced by pre-training the model, even on a different cell. This once again is evidence that pre-training contributes to minimizing the test set error regardless of the cell type. Supplementary Fig. 2 illustrates the estimation of the worst performing mode. Despite being trained on a different cell type, the model still can capture the trend of the ground truth SOC value. With pretraining on the LG cell and re-training on the Panasonic cell, model can estimate the SOC more accurately as shown in Fig. 4.

In this section, we showed that the weights of the model that is learned during the unsupervised pre-training phase can be re-used in re-training or fine-tuning across different cell types. This opens the possibilities of transfer learning which is extremely helpful especially when data and computational resource is scarce. On a side note, all re-training and fine-tuning in this and the previous section was only performed for 5 epochs to showcase the learning capabilityof the model despite a small training epoch. Re-training and fine-tuning the model for more epochs will likely yield better performance. 


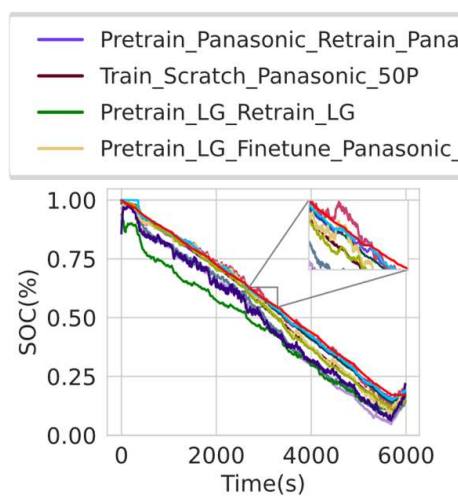

(a)

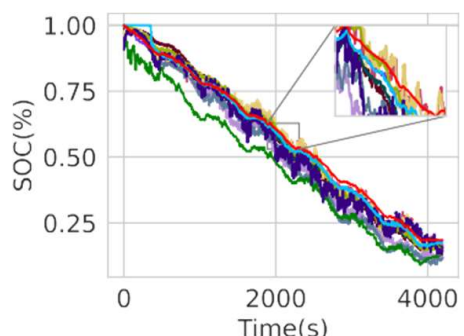

(d)

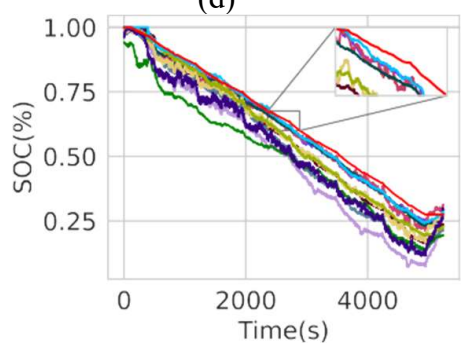

(g)

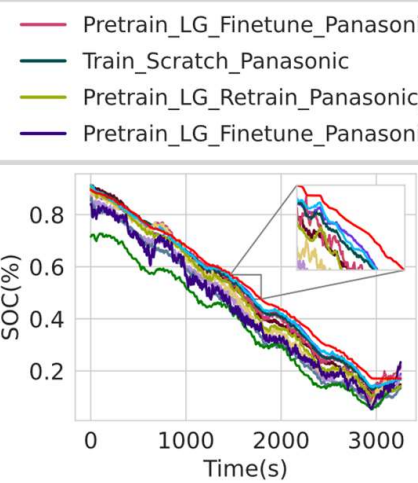

(b)

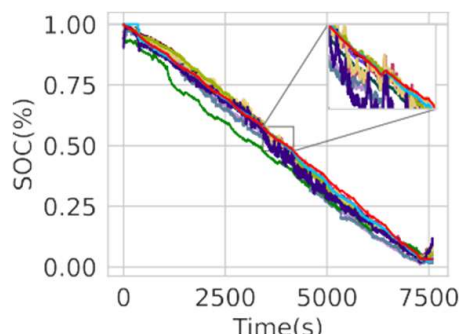

(e)

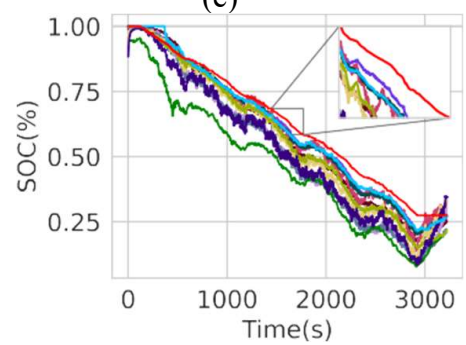

(h)

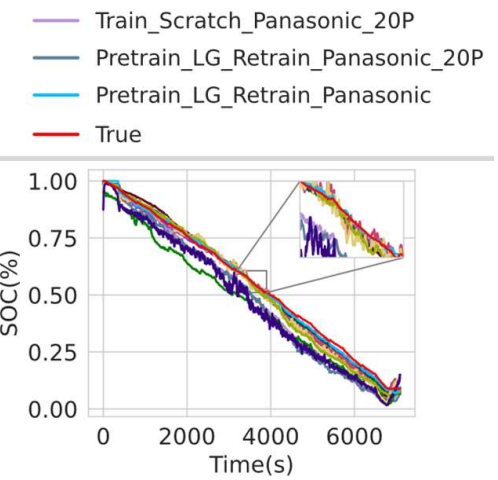

(c)

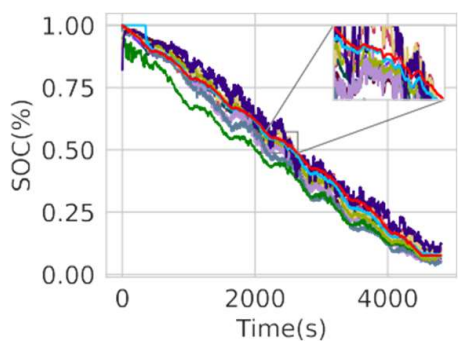

(f)

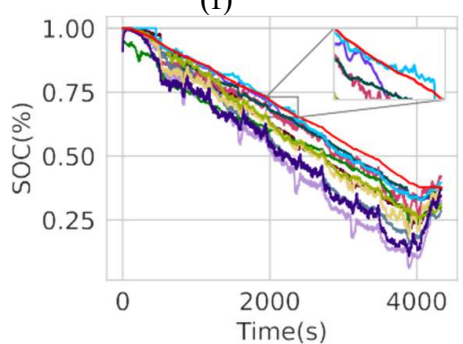

(i)

Fig. 4 Estimation plot on the test drive cycles of the Panasonic cell with various training combinations. (a) HWFET cycle at $0{ }^{\circ} \mathrm{C}$. (b) US06 cycle at $0{ }^{\circ} \mathrm{C}$. (c) HWFET cycle at $10^{\circ} \mathrm{C}$. (d) US06 cycle at $10^{\circ} \mathrm{C}$. (e) HWFET cycle at $25^{\circ} \mathrm{C}$. (f) US06 cycle at $25^{\circ} \mathrm{C}$.

\section{Discussion}

In this work, a data-efficient Transformer-based SOC estimation model in combination with the SSL framework is developed to address the challenges on Li-ion cell data availability, transfer learning, training speed and model accuracy. We showed that the proposed model can achieve the lowest RMSE and MAE on the test set at various ambient temperature settings. The proposed technique enables the model to be trained in a very short amount of time. The first contribution of this work is the introduction of a novel Transformer DL architecture that is capable of accurately estimating the SOC of a Li-ion cell under constant and varying ambient temperatures. Based on the provided dataset, the model can accurately estimate the SOC up to RMSE $\leq 1.19 \%$ and MAE $\leq 0.65 \%$ (at varying ambient temperatures) and RMSE $\leq 0.9 \%$ and MAE $\leq 0.44 \%$ (at constant ambient temperature) with no feature engineering or any type of filtering. This also shows that the transformer can directly self-learn the model parameters to map the voltage, current and temperature data directly to SOC.

The second contribution is the self-supervised learning (SSL) training scheme to effectively train the proposed model. Even though the conventional supervised learning (SL) scheme can train the proposed model up to a reasonably low error (RMSE $\leq 1.63 \%$ at varying ambient temperatures), this work highlights that the SSL training framework is advantageous in further reduction of error rate (RMSE $\leq 1.42 \%$ at varying ambient temperatures) at approximately the same amount of training time. This suggests that the SSL framework proposed to train the transformer contributes to lowering the RMSE. 
The third contribution of this work is to demonstrate that the weights from the encoder layers of the transformer learned using the unsupervised pre-training phase can be readily transferred to another cell type of a different chemistry. Additionally, with only five epochs of re-training, the model can achieve RMSE $\leq 2.5 \%$ on the test set. Extending the training time further likely leads to further reduction in RMSE. However, this work shows that even with lightweight retraining, the weights transferred from pre-training significantly contribute to the short training time with significantly less data. This opens the possibility of adapting the Transformer model to other types of Li-ion cell with only a fraction of the training data.

The fourth contribution highlights the SSL training framework that enables the proposed model to be re-trained with as few as $20 \%$ of the total training data and still achieve lower error compared to the models that do not use the SSL framework. Despite the reduced amount of training data, the model still generalizes well across different cell chemistry. This further accentuates the important role of unsupervised pretraining in allowing the model to be more data efficient.

The fifth contribution compares and validates the performance of the model against recent state-of-the-art DL models on SOC estimation. It is shown that the model clearly outperforms all other models in the RMSE and MAE metric on the test dataset. Given the efficacy of the model in SOC estimation accuracy, transfer learning capability, dataefficiency and training speed, the proposed Transformer model and framework is evidently advantageous over other DL models.

\section{Methods}

Dataset. In this study, we utilized raw data sampled from a brand-new cylindrical $18650 \mathrm{LiNiMnCoO} 2$ cell by LG which was made available by the McMaster University in Hamilton, Ontario, Canada ${ }^{45}$. The specification of the cell is given in Supplementary Table 1. The data was collected by subjecting the battery cell to various EV standard drive cycles such as UDDS, HWFET, LA92, US06 at varying ambient temperatures ranging from $-20^{\circ} \mathrm{C}$ to $40^{\circ} \mathrm{C}$. In addition, to simulate the dynamics of driving conditions, the cell was also subjected to a random mix of the standard drive cycles. The division of train, validation and test dataset used in this study is specified in Supplementary Table 3. Fig. 5(a) illustrates a sample plot of the UDDS drive cycle from the test dataset at $-20^{\circ} \mathrm{C}$ ambient temperature. For DL models to work well, careful consideration is put in preprocessing the raw data samples. Firstly, the raw data is normalized into a range of 0 to 1 using Equation 1.

$$
\tilde{x}=\frac{x-\min (x)}{\max (x)-\min (x)}
$$

Next, the raw data is divided into three separate sets namely train, validation, and test set. The train set is used to train the model, validation set to check the generalization of the model during training and the test set is only used to evaluate the model at the end of training. The division of the train, valid and test set is tabulated in Supplementary Table 3.

Using supervised learning requires the dataset to be formatted into a input-label pairs. In this study the input is the normalized values of voltage, current, and temperature while the label corresponds to the SOC value. The inputlabel pairs was constructed by running a sliding window across the time axis over the train, validation, and test set as illustrated Fig. 5. Voltage, current and temperature values that resides in the green window corresponds to the input and SOC value that resides in the purple window corresponds to the label. Note that the width of the window, $k$ is set to $k=400$ timesteps.

Having massive amounts of data is a crucial component in training the proposed model without which the model will fail to generalize well ${ }^{46}$. Although the raw data already consists of hundreds of thousands of timesteps, it is still insufficient for the proposed model to work. Hence, the original dataset was augmented in various way by injecting random noise $(\mu=0, \sigma=0.33)$ onto the training and validation dataset. The types of noise injected includes additive and multiplicative Gaussian noise on the magnitude and random frequency noise generated with the wavelet decomposition method. Fig. 5(b) shows a sample of the original and the augmented version of the plot.

Transformer Model Architecture. The original Transformer proposed $\mathrm{in}^{47}$ uses the encoder-decoder arrangement in the architecture. The model proposed in this work only adopts the encoder portion and not the decoder as detailed $\mathrm{in}^{48}$ to work better with multivariate time-series data. Fig. 6 illustrates the block diagrams of the proposed model depending on the training stage which will be detailed more in the next section. Observe that in Stage 1 and Stage 2 there are several common blocks namely, input, $x$ positional encoding, encoder stack, and linear layer. The input data to the model consists of the input vector of $X=\left[V_{k}, I_{k}, T_{k}\right]$ representing the cell voltage, current and temperature at timestep, $k$. To give the input data contextual information, the input vector is then added to the positional 
encoding. There are various choices of positional encodings according to ${ }^{49}$. However, in this work we use the learned positional encoding with the sine and cosine functions as shown in Eq. 2 and Eq. 3.

$$
\begin{gathered}
P E_{(p o s, 2 i)}=\sin \left(\frac{p o s}{10000^{2 i / d_{\text {model }}}}\right) \\
P E_{(\text {pos }, 2 i+1)}=\cos \left(\frac{\text { oos }}{10000^{2 i / d_{\text {model }}}}\right)
\end{gathered}
$$

where pos and $i$ correspond to the position and dimension, respectively. The reasoning behind using both functions has been previously detailed $\mathrm{in}^{47}$. Once tagged with the positional encodings, the input vector is passed through a series of encoder blocks. Core to the Transformer architecture is the multi-headed self-attention (MHSA)module inside the encoder block. The MHSA module applies self-attention to the input sequencewith respect to the output sequence. As shown in the figure, the input to the multi-headed self- attention module are the key, $K$, value, $V$ and query, $Q$. In the MHSA block, it attempts to map the query to a set key-value pairs with respect to an output to produce the attention matrix. The operation consists of a dot product of the query, Q with all keys and a division by $d_{k}$ and applying a softmax function over the result as given in Eq. 4 where $\mathrm{dk}$ is the dimension of the keys.
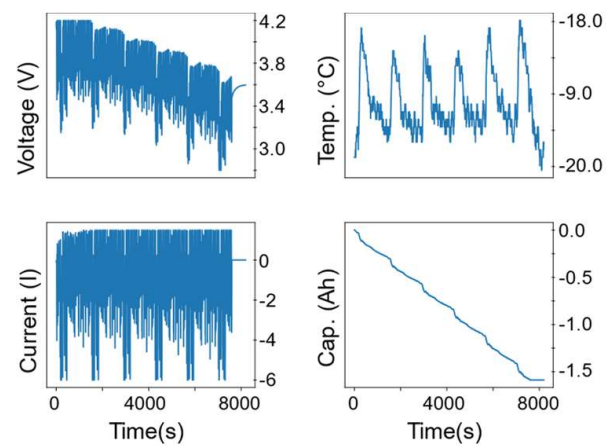

(a)

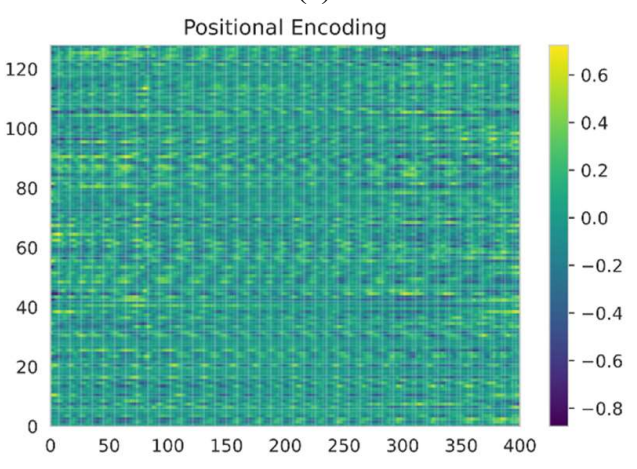

(c)

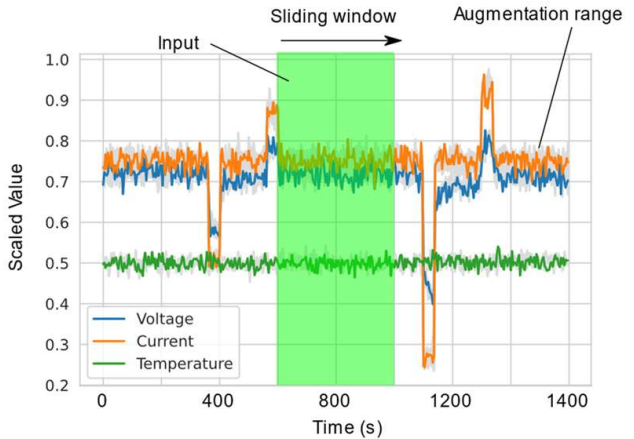

(b)

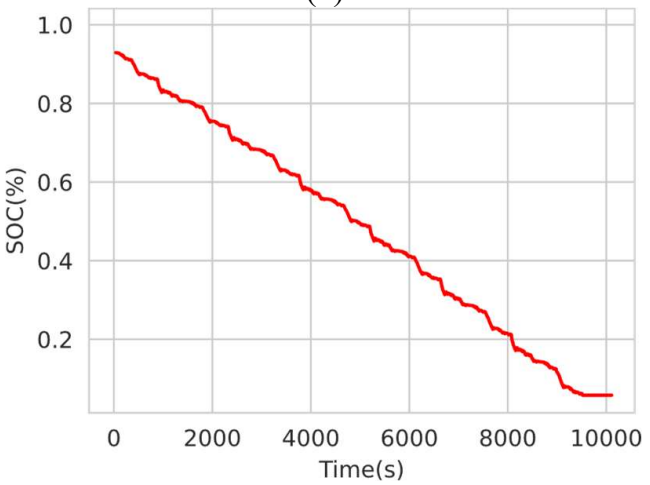

(d)

Fig. 5 Data preprocessing pipeline from raw values to positional encoding. (a) Raw data sampleplot from the UDDS drive cycle at $-20^{\circ} \mathrm{C}$ ambient temperature. (b) Input-label pairs constructed by sliding a fix width window across the train, validation, and test dataset. (c) Dataset converted into positional encoding to be ingested into Transformer.

$$
\operatorname{Attention}(Q, K, V)=\operatorname{softmax}\left(\frac{Q K^{T}}{\sqrt{d_{k}}}\right) V
$$

Instead of performing the operation in Eq. 4 once to produce a single matrix, the operationcan be repeated multiple times in parallel and the resulting matrices can be concatenated into a larger matrix as show in Eq. 5 .

$$
\operatorname{MultiHead}(Q, K, V)=\operatorname{Concat}\left(\text { Attention }_{1}, \text { Attention }_{2} \ldots \text { Attention }_{n}\right) W^{O}
$$

In this work we use the number of parallel attention heads, $h=16$. Each head uses $d_{\text {model }}=128$. Also used in the encoder module is the residual connection and residual dropout, which was set at dropout probability, $p=0.1$. The remaining model hyperparameter values are in Supplementary Table 4. Since Transformer models processes the entire sequence and does not account for the order of the input, a positional encoder is required to 
add contextual information. The positional encoder generates a unique encoding for each data point in the input vector and can generalize to longer sequences. Shown in Fig. 5(c) is the learned positional encoding generated for the input dataset in this study which consists of voltage, current and temperature of the Li-ion cell. The $\mathrm{x}$-axis corresponds to the lag window in the input dataset which is used generate the dataset.

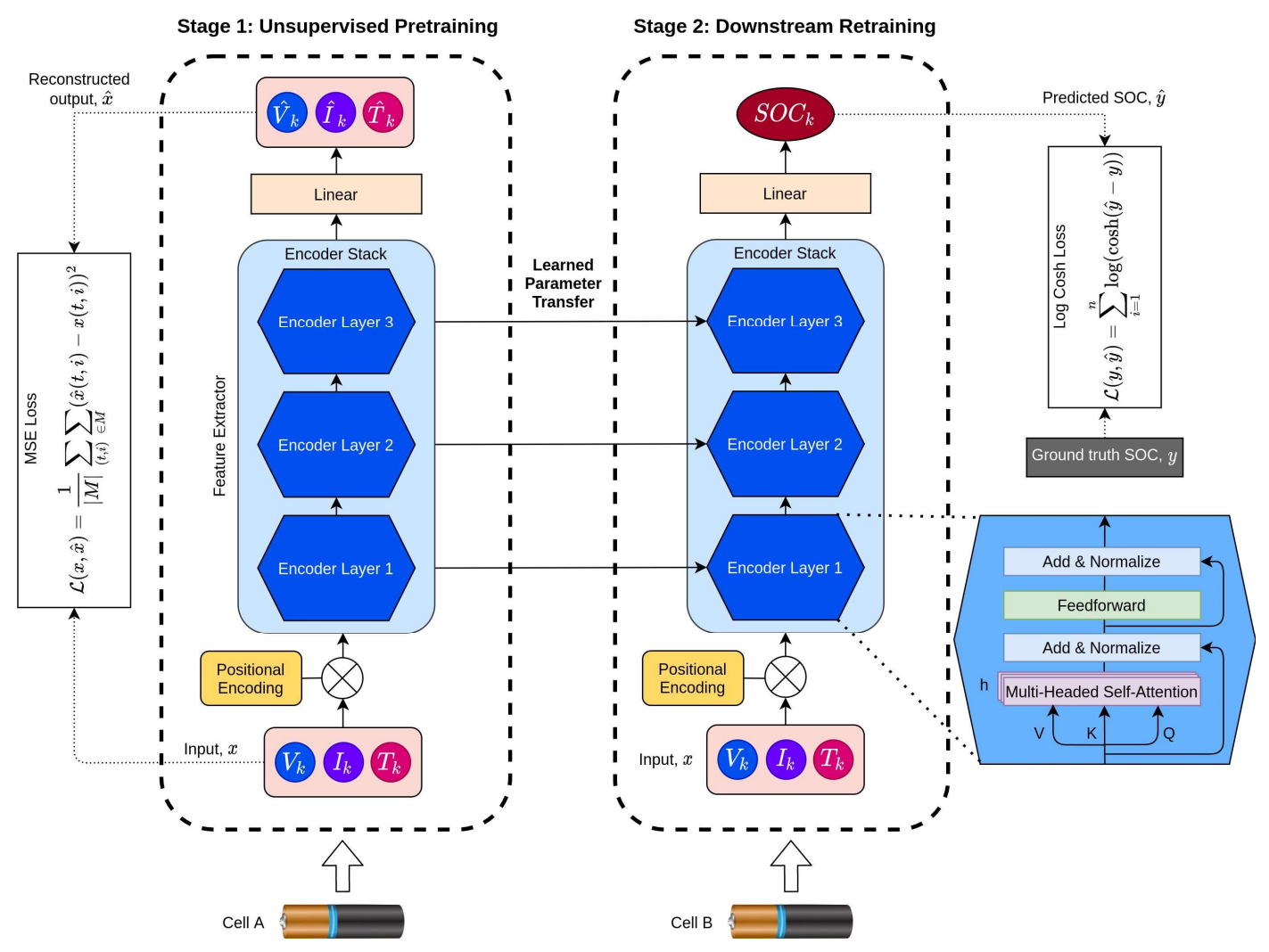

Fig. 6 Framework and architecture of the proposed transformer model.

Training Framework. Instantiating a DL model involves various stochastic płocess. To ensure the reproducibility and consistency of the results obtained, all experiments were conducted using a preset seed value. Referring to Fig. 6, model training was divided into two distinct phases, namely the unsupervised pre-training and downstream fine-tuning. In the unsupervised pre-training stage ${ }^{50}$, unlabeled vectors of input sequence, $\mathrm{X}$ was used to train the model. Part of each input sequence values were randomly set to 0 by performing element-wise multiplication with a binary mask, $M$. The corrupted input, $\mathrm{X}^{\sim}$ was generated with the $\mathrm{X}^{\sim}=\mathrm{M} 0 \mathrm{X}$. The model was then required to reconstruct the masked input with a modified MSE loss function, as given in Equation $\underline{6}$.

$$
\mathcal{L}(x, \hat{x})=\frac{1}{|M|} \sum_{(t, i)} \sum_{\in M}(\hat{x}(t, i)-x(t, i))^{2}
$$

where $x \hat{t}$ is the predicted input vector values and $x$ is the un-corrupted input vector values. Note that the loss does not require the model to reconstruct the entire input sequence but only elements in the mask, $M$. Upon completion of the unsupervised pre-training phase, the weights of the model save were transferred for the downstream finetuning phase. In this phase, the model was re-trained on a labeled dataset with supervised learning. The loss function, used in this phase is the hyperbolic cosine (Log-cosh) loss function as given in Equation 7.

$$
\mathcal{L}(y, \hat{y})=\sum_{i=1}^{n} \log (\cosh (\hat{y}-y))
$$

where $y$ is the ground truth and $\hat{y}$ is the predicted value by the model. The RMSE (Equation 8 ) and MAE (Equation 9) error metric was used to evaluate all models. 


$$
\begin{aligned}
& \text { RMSE }=\sqrt{\frac{1}{\mathrm{~N}} \sum_{\mathrm{k}=1}^{\mathrm{N}}\left(\mathrm{SOC}_{\mathrm{k}}-\mathrm{SOC}_{\mathrm{k}}^{*}\right)^{2}} \\
& M A E=\frac{1}{N} \sum_{k=1}^{N}\left(\left|S O C_{k}-S O C_{k}^{*}\right|\right)
\end{aligned}
$$

One of the most important hyperparameter used in training DL models is the learning rate, $\alpha$ (LR) ${ }^{51}$. In order to search for the optimal LR range of values, we employ the use of LR finder introduced in ${ }^{52}$. The optimal LR found with the LR finder is $\alpha=1 e 3$ as depicted in supplementary Fig. 1. The LR value was used in conjunction with the Ranger optimizer which is a synergistic combination of Rectified Adam (RAdam) ${ }^{53}$ and Lookahead optimizer $^{54}$. RAdamhas been shown to stabilize the training at the start and Ranger stabilizes the convergence inthe remaining step ${ }^{55}$. The Ranger optimizer is configured with momentum $=0.95$, weight decay $=0.01$ and epsilon of 1e-6. This combination has been shown to achieve state-of-the-art results on many datasets ${ }^{5-57}$. As the training approaches the end, the LR is decayed to a lower value to further facilitate convergence to a global minima ${ }^{58}$. The LR is decayed for each batch as follows,

$$
\eta_{t}=\eta_{\text {min }}^{i}+\frac{1}{2}\left(\eta_{\text {max }}^{i}-\eta_{\text {min }}^{i}\right)\left(1+\cos \left(\frac{T_{\text {current }}}{T_{i}} \pi\right)\right)
$$

$\eta$ is the maximum and minimum LR values, and $T_{\text {current }}$ is the number of epochs since the last restart. Figure shows the LR values throughout the training. The training hyperparameter values of the proposed Transformer model is concisely summarized in Supplementary Table 5.

Implementation. All models studied were trained on an Ubuntu 20.04.02 LTS Linux operating system with Intel Core $17-4790 \mathrm{~K}$ CPU at $4.00 \mathrm{GHz}$ clock frequency, $32 \mathrm{~GB}$ of RAM and a Nvidia GeForce RTX3090 graphic processing unit. All DL models were built using the open source Pytorch 1.7.159 framework in tandem with the TSAI library ${ }^{60}$. The implementation of the proposed Transformer model and SSL training framework was divided into several steps. In Step 1, two distinct datasets from the LG LiNiMnCoO2 cell (Supplementary Table 1) and Panasonic $\mathrm{LiNoCoAlO}_{2}$ cell (Supplementary Table 2) were downloaded. Both dataset consist of data sampled from respective cells over a diverse range of temperature and drive cycles to simulate dynamic operating conditions as elaborated in Section 3.1. The dataset was divided into train, validation and test sets as shown in Supplementary Table 3. Next the data was normalized into the appropriate range ( 0 to 1$)$ and pre-processed with sliding window of lag, $k=400$ timesteps (Fig. 5(b)). The sliding window lag value, $n$ is arbitrarily selected to due to the limits inour computing resources. Given more computational resources, $k$ can be made larger to allow the model to consider more contextual information from the past. At this point the dataset was also augmented by injecting additive and multiplicative Gaussian noise. Finally, the dataset is transformed into the positional encoding form shown in Fig. 5(c). This is the format of the data that is expected by the transformer model.

In Step 2, the Transformer was configured using the appropriate model hyperparameters as detailed in Supplementary Table 4. Careful attention is placed on the dropout hyperparameter value as it largely influences the degree of overfitting on the dataset. We find that the settings of dropout in the feedforward layer to $p=0.2$ and dropout in the residual layer to $p=0.1$ work well in our experiments.

In Step 3, the model is now ready to be trained. As illustrated in Section 3.3, the model was trained in two distinct stages in the order of unsupervised pretraining and then downstream retraining. In section 2.1, the model was trained on the LG dataset and was evaluated on its estimation accuracy at fixed and variable ambient temperature settings. In section 2.2, the modelwas trained on the LG dataset and tested on its estimation accuracy on the Panasonic dataset. In this step, the training hyperparameter was configured as detailed in Supplementary Table 5. Careful attention was placed on setting the LR in both training phase as it largely determines the performance and convergence to a global minimal. As detailed in section 3.3, we rely extensively on the use of a LR finder algorithm which points us to setting the LR, $\alpha=1 \times 10^{-3}$ for pretraining and $\alpha=2 \times 10^{-4}$ for retraining.

In Step 4 the model was evaluated on the SOC estimation accuracy in Section 2.1 and the influence of pretraining and SSL on the performance of the proposed model in Section 2.2. The performance of the model was quantified with the RMSE and MAE performance metrics. Finally, the performance of the model is compared to various widely used DL models on similar performance metrics. 
Data availability. The data that support the findings of this study are available from the corresponding author upon reasonable request.

Code availability. The software code and the examined cases that validated our method are available from the corresponding author upon reasonable request.

\section{References}

1. EPA (Environmental Protection Agency), USA. Sources of Greenhouse Gas Emissions. https://www.epa.gov/ghgemissions/sources-greenhouse-gas-emissions.

2. Luisa, M., Silvestre, D., Favuzza, S., Sanseverino, E. R. \& Zizzo, G. How Decarbonization , Digitalization and Decentralization are changing key power infrastructures. Renew. Sustain. Energy Rev. 93, 483-498 (2018).

3. Teoh, T., Kunze, O., Teo, C. C. \& Wong, Y. D. Decarbonisation of urban freight transport using electric vehicles and opportunity charging. Sustain. 10, (2018).

4. Berckmans, G. et al. Cost projection of state of the art lithium-ion batteries for electric vehicles up to 2030. Energies 10, 1-20 (2017).

5. Ilott, A. J., Mohammadi, M., Schauerman, C. M., Ganter, M. J. \& Jerschow, A. Rechargeable lithium-ion cell state of charge and defect detection by in-situ inside-out magnetic resonance imaging. Nat. Commun. 9, 1776 (2018).

6. Schmuch, R., Wagner, R., Hörpel, G., Placke, T. \& Winter, M. Performance and cost of materials for lithium-based rechargeable automotive batteries. Nat. Energy 3, 267-278 (2018).

7. Hannan, M. A. et al. Toward Enhanced State of Charge Estimation of Lithium-ion Batteries Using Optimized Machine Learning Techniques. Sci. Rep. 10, 4687 (2020).

8. How, D. N. T. et al. State of Charge Estimation for Lithium-ion Batteries Using Model-Based and Data-Driven Methods: A review. IEEE Access 7, 136116-136136 (2019).

9. Shrivastava, P., Soon, T. K., Idris, M. Y. I. Bin \& Mekhilef, S. Overview of model-based online state-of-charge estimation using Kalman filter family for lithium-ion batteries. Renewable and Sustainable Energy Reviews vol. 113109233 (2019).

10. Fleischer, C., Waag, W., Bai, Z. \& Sauer, D. U. Self-learning state-of-available-power prediction for lithium-ion batteries in electrical vehicles. in IEEE Vehicle Power and Propulsion Conference 370-375 (2012).

11. Kim, W. Y., Lee, P. Y., Kim, J. \& Kim, K. S. A nonlinear-model-based observer for a state-of-charge estimation of a lithium-ion battery in electric vehicles. Energies 12, 1-20 (2019).

12. Ozcan, G. et al. Online state of charge estimation for Lithium-ion batteries using Gaussian process regression. in IECON Proceedings 998-1003 (2016).

13. Han, X., Ouyang, M., Lu, L. \& Li, J. Simplification of physics-based electrochemical model for lithium ion battery on electric vehicle. Part II: Pseudo-two-dimensional model simplification and state of charge estimation. J. Power Sources 278, 814-825 (2015).

14. Lipu, M. S. H. et al. Data-driven state of charge estimation of lithium-ion batteries: Algorithms, implementation factors, limitations and future trends. J. Clean. Prod. 277, 124110 (2020).

15. Liao, L. \& Köttig, F. A hybrid framework combining data-driven and model-based methods for system remaining useful life prediction. Appl. Soft Comput. J. 44, 191-199 (2016).

16. Deng, Z. et al. Data-driven state of charge estimation for lithium-ion battery packs based on Gaussian process regression. Energy 205, 118000 (2020).

17. Lipu, M. S. H. et al. Intelligent algorithms and control strategies for battery management system in electric vehicles: Progress, challenges and future outlook. J. Clean. Prod. 292, 126044 (2021).

18. How, D. N. T. et al. State-of-Charge Estimation of Li-Ion Battery in Electric Vehicles: A Deep Neural Network Approach. IEEE Trans. Ind. Appl. 56, 5565-5574 (2020).

19. Hannan, M. A. et al. SOC Estimation of Li-ion Batteries with Learning Rate-Optimized Deep Fully Convolutional Network. IEEE Trans. Power Electron. 36, 7349-7353 (2021).

20. Hannan, M. A. et al. State-of-Charge Estimation of Li-ion Battery Using Gated Recurrent Unit with One-Cycle Learning Rate Policy. IEEE Trans. Ind. Appl. 57, 2964-2971 (2021).

21. Chemali, E., Kollmeyer, P. J., Preindl, M., Ahmed, R. \& Emadi, A. Long Short-Term Memory Networks for Accurate State-of-Charge Estimation of Li-ion Batteries. IEEE Trans. Ind. Electron. 65, 6730-6739 (2018).

22. Chemali, E., Kollmeyer, P. J., Preindl, M. \& Emadi, A. State-of-charge estimation of Li-ion batteries using deep neural networks: A machine learning approach. J. Power Sources 400, 242-255 (2018).

23. Yang, F., Li, W., Li, C. \& Miao, Q. State-of-charge estimation of lithium-ion batteries based on gated recurrent neural network. Energy 175, 66-75 (2019).

24. Huang, Z., Yang, F., Xu, F., Song, X. \& Tsui, K.-L. Convolutional Gated Recurrent Unit-Recurrent Neural Network for State-of-Charge Estimation of Lithium-Ion Batteries. IEEE Access 7, 93139-93149 (2019).

25. Zhang, Z. et al. An Improved Bidirectional Gated Recurrent Unit Method for Accurate State-of-Charge Estimation. IEEE Access 9, 11252-11263 (2021). 
26. Xiao, B., Liu, Y. \& Xiao, B. Accurate state-of-charge estimation approach for lithium-ion batteries by gated recurrent unit with ensemble optimizer. IEEE Access 7, 54192-54202 (2019).

27. Huang, Z., Yang, F., Xu, F., Song, X. \& Tsui, K. L. Convolutional Gated Recurrent Unit-Recurrent Neural Network for State-of-Charge Estimation of Lithium-Ion Batteries. IEEE Access 7, 93139-93149 (2019).

28. Song, X., Yang, F., Wang, D. \& Tsui, K. L. Combined CNN-LSTM Network for State-of-Charge Estimation of Lithium-Ion Batteries. IEEE Access 7, 88894-88902 (2019).

29. Holmberg, O. G. et al. Self-supervised retinal thickness prediction enables deep learning from unlabelled data to boost classification of diabetic retinopathy. Nat. Mach. Intell. 2, 719-726 (2020).

30. Bhattacharjee, A., Verma, A., Mishra, S. \& Saha, T. K. Estimating State of Charge for xEV Batteries Using 1D Convolutional Neural Networks and Transfer Learning. IEEE Trans. Veh. Technol. 70, 3123-3135 (2021).

31. Ren, X., Liu, S., Yu, X. \& Dong, X. A method for state-of-charge estimation of lithium-ion batteries based on PSOLSTM. Energy 234, 121236 (2021).

32. Lipu, M. S. H. et al. State of Charge Estimation for Lithium-ion Battery Using Recurrent NARX Neural Network Model Based Lighting Search Algorithm. IEEE Access 6, 28150-28161 (2018).

33. Li, S. et al. State-of-Charge Estimation of Lithium-Ion Batteries in the Battery Degradation Process Based on Recurrent Neural Network. Energies 14, 306 (2021).

34. Bazi, Y., Bashmal, L., Al Rahhal, M. M., Dayil, R. Al \& Ajlan, N. Al. Vision transformers for remote sensing image classification. Remote Sens. 13, 1-20 (2021).

35. Popel, M. et al. Transforming machine translation: a deep learning system reaches news translation quality comparable to human professionals. Nat. Commun. 11, 1-15 (2020).

36. Tetko, I. V., Karpov, P., Van Deursen, R. \& Godin, G. State-of-the-art augmented NLP transformer models for direct and single-step retrosynthesis. Nat. Commun. 11, 1-11 (2020).

37. Eun, D. in et al. Deep-learning-based image quality enhancement of compressed sensing magnetic resonance imaging of vessel wall: comparison of self-supervised and unsupervised approaches. Sci. Rep. 10, 1-17 (2020).

38. He, K., Zhang, X., Ren, S. \& Sun, J. Deep residual learning for image recognition. in Proceedings of the IEEE Computer Society Conference on Computer Vision and Pattern Recognition 770-778 (2016).

39. Weimann, K. \& Conrad, T. O. F. Transfer learning for ECG classification. Sci. Rep. 11, 1-12 (2021).

40. Ismail Fawaz, H. et al. InceptionTime: Finding AlexNet for time series classification. Data Min. Knowl. Discov. 34, 1936-1962 (2020).

41. Wang, Z., Yan, W. \& Oates, T. Time series classification from scratch with deep neural networks: A strong baseline. in International Joint Conference on Neural Networks 1578-1585 (2017).

42. Jiao, M., Wang, D. \& Qiu, J. A GRU-RNN based momentum optimized algorithm for SOC estimation. J. Power Sources 459, 228051 (2020).

43. Wei, M., Ye, M., Li, J. B., Wang, Q. \& Xu, X. State of Charge Estimation of Lithium-Ion Batteries Using LSTM and NARX Neural Networks. IEEE Access 8, 189236-189245 (2020).

44. Wu, J., Li, T., Zhang, H., Lei, Y. \& Zhou, G. Research on modeling and SOC estimation of lithium iron phosphate battery at low temperature. Energy Procedia 556-561 (2018).

45. Vidal, C. et al. Robust xEV Battery State-of-Charge Estimator Design Using a Feedforward Deep Neural Network. SAE Tech. Pap. 2020-April, (2020).

46. Goodfellow, I., Bengio, Y. \& Courville, A. Deep Learning. (MIT press Cambridge, 2020).

47. Vaswani, A. et al. Attention is all you need. Adv. Neural Inf. Process. Syst. 5999-6009 (2017).

48. Zerveas, G., Jayaraman, S., Patel, D., Bhamidipaty, A. \& Eickhoff, C. A Transformer-based Framework for Multivariate Time Series Representation Learning. (2020).

49. Gehring, J., Auli, M., Grangier, D., Yarats, D. \& Dauphin, Y. N. Convolutional sequence to sequence learning. in International Conference on Machine Learning vol. 3 2029-2042 (2017).

50. Caron, M., Bojanowski, P., Mairal, J. \& Joulin, A. Unsupervised Pre-Training of Image Features on Non-Curated Data. Proc. IEEE Int. Conf. Comput. Vis. 2019-October, 2959-2968 (2019).

51. Li, Z., Lyu, K. \& Arora, S. Reconciling Modern Deep Learning with Traditional Optimization Analyses: The Intrinsic Learning Rate. (2020).

52. Smith, L. N. Cyclical Learning Rates for Training Neural Networks. IEEE Winter Conf. Appl. Comput. Vis. 464472 (2015).

53. Liu, L. et al. On the Variance of the Adaptive Learning Rate and Beyond. (2019).

54. Zhang, M. R., Lucas, J., Hinton, G. \& Ba, J. Lookahead Optimizer: k steps forward, 1 step back. Adv. Neural Inf. Process. Syst. 32, (2019).

55. Valeri, J. A. et al. Sequence-to-function deep learning frameworks for engineered riboregulators. Nat. Commun. 11, 1-14 (2020).

56. Zhang, P., Yang, L. \& Li, D. EfficientNet-B4-Ranger: A novel method for greenhouse cucumber disease recognition under natural complex environment. Comput. Electron. Agric. 176, 105652 (2020).

57. Bao, Y. et al. Named entity recognition in aircraft design field based on deep learning. in Lecture Notes in Computer Science (including subseries Lecture Notes in Artificial Intelligence and Lecture Notes in Bioinformatics) vol. 12432 LNCS 333-340 (Springer Science and Business Media, 2020). 
58. Loshchilov, I. \& Hutter, F. SGDR: Stochastic Gradient Descent with Warm Restarts. in International Conference on Learning Representations 1-6 (2016).

59. Paszke, A. et al. PyTorch: An Imperative Style, High-Performance Deep Learning Library. Adv. Neural Inf. Process. Syst. 32, (2019).

60. Torres, J. F., Hadjout, D., Sebaa, A., Martínez-Álvarez, F. \& Troncoso, A. Deep Learning for Time Series Forecasting: A Survey. Big Data 9, 3-21 (2021).

\section{Acknowledgements}

This work was supported by the LRGS project grant number 20190101LRGS from the Ministry of Higher Education, Malaysia under Universiti Tenaga Nasional and in part by the UNITEN Bold Refresh Publication Fund 2021 under project J5100D4103 and partial support by ARC Research Hub for Integrated Energy Storage Solutions, UNSW.

\section{Author Contributions}

M.A.H. and D.N.T.H. designed the research. D.N.T.H., M.A.H., M.S.H.L., K.P.J. and M.M. conducted the machine learning optimization and modelling, performed the experiments, analyzed the data and wrote the manuscript. Z.Y.D., K.S.M.S., S.K.T., K.M.M, T.M.I.M., and F.B. provided study oversight and edited the manuscript. All authors discussed the results and commented on the manuscript.

\section{Competing Interests}

The authors declare no competing interests. 


\section{Supplementary Files}

This is a list of supplementary files associated with this preprint. Click to download.

- SupplementaryInformation.docx 